\section{Reworking the Science of Adam}

\author{
S. Joshua Swamidass (D) \\ https://doi.org/10.54739/qv13
}

his last year, 2017 till now, we have been reworking the science of Adam. This brings us several new ways to think about Adam and Eve in human origins. This is significant in the conversation between science and the Christian faith, as two fairly large scientific errors have been corrected. In all these cases, it is in critique of Adam and the Genome that these corrections have come into the spotlight.

There are two important developments, and one important event, to report:

1. An article explaining the science of universal (and recent) genealogical ancestry is now available in PSCF, and on our website (free of the pay wall).

2. Questions from Dr. Richard Buggs about the genetic evidence have clarified that a sole-genetic progenitor origin may be possible if we are willing to place Adam and Eve before 500 kya.

3. This summer, at the upcoming ASA conference, on Friday, July 27 from 2 pm-5 pm, I am hosting a workshop where we will look at the developments in more detail: Reworking the Science of Adam and Eve. Please consider joining us.

There has been substantial engagement from theologians on a genealogical Adam (\#1 above). I will be presenting at a second seminary on this in April, and will be a featured paper at the Dabar Conference in June. These events, however, are not open to public, but this lays the groundwork for more important things in the future. To help support broader engagement, we have also launched a forum for Peaceful Science, which is open to the public. Join us there if you want to go deeper.

Here is a quick primer on how things have been shaping up on the scientific side.

\section{What is "Human"?}

These developments draw attention to the ambiguity of the term "human" in origins. There are several points in history identified, by different people, with the rise of "human." There is no consensus in theology or science on how to define "human," so we expect an unresolvable diversity of opinions.

1. 12 to 6 kya, when civilization and agriculture arise, then spread quickly across the globe.

2. About 70 to $50 \mathrm{kya}$, with the rise of behaviorally-modern humans.

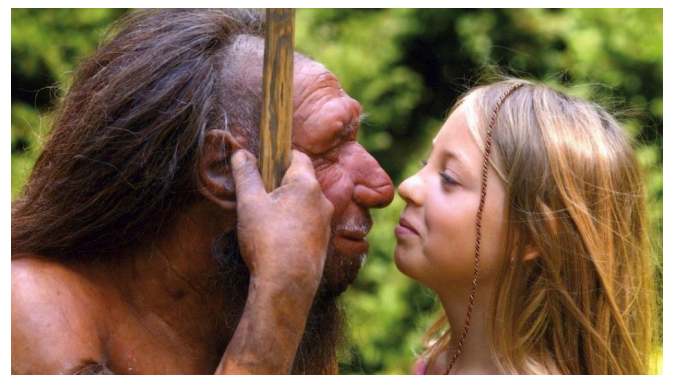

3. About 100 to $300 \mathrm{kya}$, with the rise of anatomically-modern humans, also known as Homo sapiens.

4. About 500 to $700 \mathrm{kya}$, with the common ancestor of Neanderthals, Homo sapiens, Denisovans, and likely some other hominins.

5. About 2 mya, with the rise of the first in the Homo genus.

In all these contexts, Adam and Eve could be genealogical universal ancestors. They would be a single couple, whose offspring interbreed with a larger population, perhaps de novo created, that become genealogical ancestors of everyone. If genealogical descent from them conveys an important theological status, such as original sin, they would be the sole genealogical progenitors of the "mankind" of Scripture, even though they interbreed with those outside the Garden.

Encouraged by questions from Dr. Richard Buggs, recent work suggests it also may also be possible for us all to arise, genetically, from a single couple. However, in this case, Adam would need to be placed at least before $500 \mathrm{kya}$. In these cases (options 4 or 5), Adam and Eve could be our sole genetic progenitors. However, if Adam and Eve are de novo created in these scenarios, this raises questions about why God would create them so consistent with a false history of common descent. The dust has yet to settle on the scientific details, but it looks likely at this point that a bottleneck anytime before 700 kya is undetectable in genetic data.

Of course, none of this work demonstrates that Adam exists. Rather, the evidence does not tell us one way or another. Where science is silent, theology has legitimate autonomy.

\section{Genealogical Adam Paper at PSCF}

I made a fairly radical claim at a public lecture at Urbana Seminary, then at a scientific seminar at the ASA conference, then in an online symposium for The Creation Project. This claim is consistent with the genetic evidence in which it appears (1) our ancestors arise as a population, not a single couple, and that (2) we share ancestry with the great apes.

Entirely consistent with the genetic evidence, it is possible Adam was created out of dust, and Eve out of his rib, less than 10,000 years ago in a divinely created garden where God might dwell with them, the first beings with opportunity to be in a relationship with Him. Perhaps their fall brought accountability for sin to all their descendants. Leaving the Garden, their offspring blended with their neighbors ${ }^{1}$ in the surrounding towns. In this way, they became 
genealogical ancestors of all those in recorded history. Adam and Eve, here, are the single-couple progenitors ${ }^{2}$ of all mankind. Even if this scenario is false or unnecessary, nothing in evolutionary science unsettles this story. So, evolution presses in a very limited way on our understanding of Adam and Eve, only suggesting (alongside Scripture) that their lineage was not pure.

This has been covered in detail, elsewhere. Now, however, an article justifying this claim has been published in PSCF, and is available here. The good news is that scientific debate is largely settled. In the coming months and years, we can anticipate an engaging conversation about the theological questions that are arising.

\section{A Genetic Sole Progenitor?}

In another surprising twist, it appears that the genetic evidence might be consistent with genetic sole-progenitorship of Adam and Eve if they are $500 \mathrm{kya}$, or farther back. Of course, they would not be Homo sapiens, but they might still be "human."

One new contribution to this conversation is a genome-wide measurement of the "Time to Most Recent 4 Alleles" (TMR4A). Under the reasonable starting point that Adam and Eve had biology much like ours, with four alleles at each position in the genome, we can use this to test several models of human origins against the data. The data looks, somewhat, like this:

This figure, adapted from Hayakawa et. al., shows a phylogeny constructed from human variation data at a particular place in the genome. Each leaf of the tree is a version of a gene that we see in some individuals. The tree is the inferred history of this gene, tracing back to a common genetic ancestor about 2.9 million years ago. The dots on the edits are mutations to the ancestral gene, and we can directly measure, using recently developed technology, how quickly they accrue over time. It is these mutations that tell the time at which different events in the history of the gene take place. Incorrectly, some people make the assumption that Adam and Eve had to be homozygous clones and interpret this as evidence against their existence after 2.9 million years ago. However, if they were made with genetic diversity, then we really should look at when there were four alleles (TMR4A), at about 500 thousand years ago.

We studied the TMR4A across the genome to see what an unbiased estimate, that takes recombination into account, might look like. It

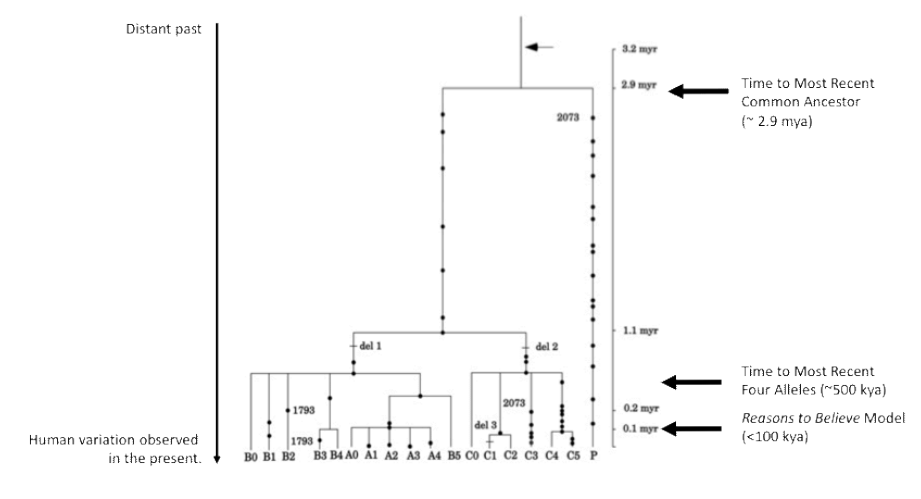

1. Their neighbors would have been created by an evolutionary process.

2. Keep in mind that sole-progenitorship does not preclude intermixing with other lines. appears that the human variation data with a single couple genetic origin, unless Adam and Eve were more than 500 thousand years ago. Taking into account other data, this might be pushed back to about 700 kya. Trans-species variation, also, does not seem to give us definitive evidence against a bottleneck of a single couple.

This figure shows the estimate of the median TMR4A at 500 thousand years ago, based on the variation in 54 unrelated individuals, of TMR4A. Note that $50 \%$ of the genome seem to be much older, either because it really is older, or because there is error in the estimate of TMR4A. In many ways, this is a very generous estimate, which might be pushed upwards with additional analysis.

\section{Testing Model's of Human Origin}

The key thing to understand is that we are using the formula: $D=2 x$ $T \times R$. Or that the number of differences (D) between two genetic samples is equal to two times the time at which their ancestry separates $(T)$ and the rate at which mutations are taking place (R). This formula is the fundamental finding of neutral theory, and applies most strongly in neutral regions of the genome. Moreover, we can directly measure mutation rates now, for the first time in history.

This gives us a way of experimentally testing different human origin models using the TMR4A. . This is a new opportunity, because we have recent been able to directly measure mutation rates across the genome (11), and it is approximately $1.25 \mathrm{e}-8$ per base pair per generation (with 25 years per generation). These models of origins seem to be in error:

1. The Reasons to Believe model requires Adam to arise before 100 kya. If TMR4A was at 100 kya, we would expect the mutation rate to be $2.6 \mathrm{e}-8$ per generation, or $5 \mathrm{x}$ more than observed.

2. The young earth model, with genetic instead of genealogical sole-proprietorship, needs Adam to arise between 15 kya to 6 kya. If TMR4A was at 6 kya, we would expect the mutation rate to be $43 e-8$ per generation, or $83 x$ more than observed.

3. Evolutionary Creationism (as put forward by BioLogos) taught that a single couple origin of all human genetics is impossible, because there was not enough time to generate the observed diversity before chimpanzees and humans diverged. For this to be true, the mutation rate would have to be at most $0.1 \mathrm{e}-8$, or $1 /$ 12th the observed mutation rate.

These models of origins, on the other hand, appear to be consistent with the data.

1. Common Descent predicts the mutation rate to be $1.65 \mathrm{e}-8$. This is number is computed using the fossil record and the observed
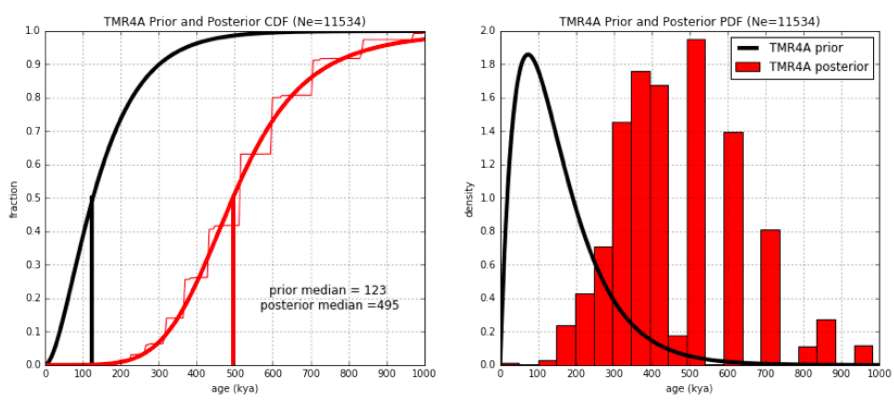
differences between humans and the great apes. It is just 1.3x the observed number, the closest of all predictions to the data.

2. Ann Gauger's Model which places a sole-genetic progenitor Adam and Eve at 2 mya, with the rise of Homo, appears consistent with the data.

So the observed TMR4A result appears to be in conflict with YEC creationism, Reasons to Believe old earth creationism, and also Evolutionary Creationism as put forward by BioLogos. However, it is consistent with both an Ancient Adam and also with Common Descent. Moreover, the data seems to validate a prediction of the Common Descent model with surprisingly high accuracy. In the associated forum topic, we will the details of this analysis as questions arise. In particular, astute readers will have several questions about alternative lines of evidence (e.g. PSMC, MSMC, introgression, trans-species variation, etc.).

\section{References}

https://peacefulscience.org/books/adam-genome/

https://www.asa3.org/ASA/PSCF/2018/

PSCF3-18p19-35Swamidass.pdf

https://peacefulscience.org/pdf/PSCF3-18p19-35Swamidass.pdf

https://discourse.peacefulscience.org/t/_/61

http://network.asa3.org/mpage/ASA2018

http://henrycenter.tiu.edu/dabar-conference

http://discourse.peacefulscience.org

https://peacefulscience.org/articles/genealogical-rapprochement/

https://peacefulscience.org/articles/reviewing-adam-and-the-genome/

\section{The Takeaways}

1. Join us in Boston at the ASA conference as we Rework the Science of Adam, July 27 from 2 pm-5 pm.

2. A genealogical Adam and Eve, ancestors of us all, is possible as recently as 10 kya in the Middle East.

3. A sole-genetic progenitor Adam and Eve is possible before about 500 kya, perhaps with the rise of the Homo genus, or the common ancestors of Homo sapiens and Neanderthals.

4. The TMR4A is a new way to look at the genetic data, creating space for some understandings of Adam, but also foreclosing others.

I also want to thank the Templeton Foundation and Greg Cootsona of the STEAM program. Their generous support, through the Inquiry to Common Ground, has made much of this work possible.

http://www.asa3.org/ASAradio/ASA2017Swamidass2.mp3

http://henrycenter.tiu.edu/2017/06/a-genealogical-adam-and-eve-inevolution

https://www.ncbi.nlm.nih.gov/pubmed/16272417

http://www.reasons.org/explore/blogs/todays-new-reason-to-believe/ $\mathrm{read} / \mathrm{tnrtb} / 2010 / 10 / 01 /$ were-they-real-the-scientific-case-foradam-and-eve

https://answersingenesis.org/bible-characters/adam-and-eve/whenwas-adam-created

http://www.reasons.org 